\title{
CAPCOM - Construtor de Arquiteturas Pedagógicas para Dinâmicas Colaborativas com Multimídia
}

\author{
Renan F. Almeida ${ }^{1}$,Orivaldo de L. Tavares ${ }^{1}$, Luiz F. Reinoso $^{1}$ \\ ${ }^{1}$ Departamento de Informática - Universidade Federal do Espírito Santo (UFES) \\ Vitória - ES - Brasil \\ ralmeida@ifes.edu.br, tavares@inf.ufes.br, luizfreinoso@gmail.com
}

\begin{abstract}
This article presents a platform to set up pedagogical architectures to promote collaborative dynamics that use multimedia created in field activities. Called CAPCOM, this new platform offers digital resources to support the development of collaborative educational activities with multimedia records made in the field. This research deals specifically with the digital resources to support collaborative dynamics to be performed after the field activity. With this platform for building educational architecture for collaborative dynamics with multimedia texts, the teacher creates according to the desired educational goal activities.
\end{abstract}

Resumo. Esse artigo apresenta uma plataforma para configurar arquiteturas pedagógicas para promover dinâmicas colaborativas que usem multimídias criados em atividades de campo. Denominada CAPCOM, essa nova plataforma oferece recursos digitais para apoiar o desenvolvimento de atividades pedagógicas colaborativas com os registros multimídias feitos em campo. Este trabalho trata especificamente dos recursos digitais para suporte à dinâmicas colaborativas a serem realizadas após a atividade de campo. Com essa plataforma para construção de arquiteturas pedagógicas para dinâmicas colaborativas com multimídias, o professor cria atividades de acordo com o objetivo pedagógico pretendido.

\section{Introdução}

As atividades desenvolvidas em espaços abertos podem apresentar diversas oportunidades de aprendizado, podendo auxiliar os participantes a visualizar ou compreender as relações que existem entre os conceitos teóricos, adquiridos em sala de aula, com os conhecimentos práticos adquiridos pela experiência, conhecido como conhecimento empírico.

Considerada uma estratégia de ensino, tirar os alunos da escola para passeios, visitas técnicas, excursões ou visitas à exposições, pode fomentar para o aprendiz a oportunidade do moldar sua aprendizagem com novos conhecimentos, ampliando assim o interesse e fortalecendo sua aprendizagem. O professor assume o papel de facilitador da aprendizagem, nessa estratégia, ao propor atividades pedagógicas motivadoras e muitas vezes inéditas para os aprendizes.

Aulas de campo devem se articular entre formação teórica e conhecimentos práticos. Elas têm como objetivo ampliar o aproveitamento dos conhecimentos adquiridos, associando das abstrações mentais aos aspectos físicos e naturais dos lugares explorados nas atividades desenvolvidas em espaços abertos. 
[Seniciato and Cavassan 2004] mostram, em um estudo feito com 97 alunos da sexta série do ensino médio, que a realização de aula de campo sobre botânica, após as aulas teóricas, foi notado ganho de aprendizagem nos conceitos estudados, chegando a um aumento de $40 \%$ no conhecimento dos alunos sobre os assuntos das aulas.

Em [Oliveira and Correia 2013] foi descrita um experiência similar onde alunos do terceiro ano do ensino médio participaram de uma atividade, em um espaço aberto, cujo o objetivo era investigar o papel da aula de campo como mecanismo facilitador no processo de ensino-aprendizagem sobre o ecossistema recifal, importante ambiente do litoral de Alagoas. Segundo [Oliveira and Correia 2013]:

"A partir dos resultados obtidos nos questionários aplicados nas duas fases, observou-se que os estudantes tiveram uma mudança considerável na aquisição de conhecimentos quando comparados os dados antes e após a aula de campo."

Por se tratar de uma atividade essencialmente colaborativa, aulas de campo apresentam diversas oportunidades de aprendizado e grandes desafios associados a essa estratégia de ensino, dentre os desafios dessa atividade estão: gerenciamento dos conhecimentos adquiridos, fixação desses conhecimentos, socialização das informações registradas, gerenciamento das atividades conduzidas, delimitação do espaço a ser explorado, monitoramento do percurso feito pelos participantes, entre outros elementos.

Usando tecnologias que permitem explorar e registrar dados do mundo físico e processar esses dados, as aulas tornam-se substancialmente mais dinâmicas e motivantes, de modo a permitir experiências reais e virtuais com os dados produzidos durante uma aula de campo, proporcionando a aprendizagem colaborativa.

Segundo [Chagas 2002], desenvolver atividades colaborativas é a "condição necessária para que as redes de aprendizagem se constituam e se mantenham, em prol de concretizar a construção de conhecimento pelos seus intervenientes".

Segundo [Vieira and Tavares 2015] a flexibilidade dos ambientes virtuais colaborativos ainda não está condizente com a complexidade resultante das variações de cenário para cooperação. [Menezes et al. 2008] evidenciam, para sustentar as práticas pedagógicas, a dificuldade dos ambientes virtuais colaborativos para atender a implantação de novas propostas pedagógicas.

Este trabalho apresenta os resultados obtidos com a associação e implementação dos conceitos de Arquitetura Pedagógica (AP) [Carvalho et al. 2005] e os conceitos que fundamentam o MOrFEU (Multi Organizador Flexível de Espaços Virtuais) [Menezes et al. 2008] cujo principal objetivo é libertar o professor da complexidade da tecnologia digital necessária para sustentar as prática pedagógicas.

Segundo [Reinoso and Tavares 2015], uma Arquitetura Pedagógica é constituída de: 1. objetivo pedagógico (o que aprender); 2. atividade(s) pedagógica(s) (o que fazer para aprender); 3 metodologia (como fazer cada atividade); 4. recursos digitais (recursos disponíveis para apoiar o desenvolvimento de cada atividade). A pesquisa desenvolvida por [Vieira et al. 2011] verificou a necessidade de requisitos de software para groupwa- 
res, classificados como Learning Management Systems (LMS), mais aderentes às necessidades de cada groupwares e que favoreça a criação de APs, com maior articulação e coordenação das atividades colaborativas.

Como resultado da combinação dos conceitos supracitados, este artigo propõe um Construtor de Arquiteturas Pedagógicas para Dinâmicas Colaborativas com Multimídia, onde o professor possa descrever e criar atividades pedagógicas, de modo flexível, para que o aprendiz possa revisitar os registros feitos em um aula de campo, para realizar colaborativamente operações sobre eles, tais como classificá-los segundo o objeto ou o assunto do registro, ou compará-los com registros feito por colegas, de modo a fazer uma metareflexão sobre os eventos vivenciados na atividade em campo.

Para modelar a plataforma CAPCOM foram incorporados os conceitos criados para o projeto MOrFEu tais como Veículo de Comunicação (VCom), Unidade de Produção Intelectual (UPI) e Template para VCom, de modo a permitir que as Arquiteturas Pedagógicas, criadas pelo professor, possam ser instrumentadas com recursos digitais configurados sob medida para elas.

Esse trabalho expande a proposta apresentada em [Pereira et al. 2015], e apresenta o projeto e implementação de um espaço virtual flexível e colaborativo para criação e uso de APCs (arquiteturas pedagógicas colaborativas) com registros multimídia de atividades de campo.

\section{Ferramentas usadas pela plataforma CAPCOM}

A Google oferece serviços de processamento de imagem com georreferência, necessários para aplicações como as que podem ser geradas pela plataforma CAPCOM. No desenvolvimento do CAPCOM, foram usadas duas soluções do Google: Google Street View e o Google Maps.

O Google Street View é constituído do Google Maps, um recurso para editoração e criação de mapas geográficos e o Google Earth, uma ferramenta para navegação em mapas do globo terrestre. Apesar de integradas funcionam de forma independente, utilizando como base as posições de latitude e longitude. O recurso disponibiliza vista panorâmica de $360^{\circ}$ na horizontal e $290^{\circ}$ na vertical, permite que os usuários visualizem partes de algum lugar no mundo, que esteja mapeado na plataforma, ao nível do chão. A API do Google Street View é utilizada para visualizar e editar mídias do tipo foto esférica.

O Google Maps é um serviço gratuito para visualizar e interagir com mapas e imagens de satélite da terra. Com as implementações desse recurso é possível traçar rotas nos mapas, fazer marcações, visualizar estruturas geográficas etc. A API do Google Maps é utilizada para visualizar o contexto onde as mídias foram produzidas e visualizar mídias do tipo ponto no mapa.

Para o desenvolvimento da aplicação web foi utilizado a API do mindmeister, HTML, CSS, Java Script e PHP, a framework Yii, que é repleta de recursos digitais para desenvolvimento orientado a objetos e padrão MVC (Model-View-Controller).

\section{CAPCOM e as Práticas Pedagógicas}

No contexto contemporâneo, as teorias relacionadas ao aprendizado são normalmente referidas como teorias de aprendizagem construtivista, com ênfase na aprendizagem ativa 
e autorregulada [Lei et al. 2009]. Tal abordagem salienta a proposta dos experimentos nos quais o conhecimento não é transmitido, mas construído nas atividade ou interações sociais.

As teorias construtivistas de aprendizagem, têm um impacto significativo sobre as práticas pedagógicas, especialmente na promoção da aprendizagem ativa. Nesse contexto as teorias construtivistas solicitam diferentes ambientes de aprendizagem para suportar os elementos que fundamentam essa teoria.

Segundo [Lei et al. 2009] os ambientes construtivistas são complexos, realistas e significativos. Em um ambiente de aprendizagem complexo, o aprendiz é desafiado a construir novos conhecimentos que possam ser aplicados, de preferência no dia a dia, em situações concretas. Tal definição tem o potencial de tornar a aprendizagem uma experiência significativa pessoal, elemento esse que contribui para motivação dos aprendizes no processo de aprendizagem. A motivação, por sua vez, é um elemento essencial para alcançar um nível mais profundo de aprendizagem.

Fundamentado na teoria do construtivismo, o CAPCOM adota o paradigma AP (arquitetura pedagógica), que apoia-se na pedagogia da incerteza, pressupondo que o conhecimento nasce do movimento da dúvida, da incerteza, da busca de novas alternativas, do debate e da troca [Carvalho et al. 2005]. Buscando se diferenciar do ensino convencional de sala de aula e da materialização de apostilas de cursos para a educação à distância, uma AP abrange processos para a criação de novidades, descobertas e experimentações, centrada no ponto de vista do aprendiz, o protagonista da aprendizagem, durante a autoria de soluções individuais ou coletivas [Menezes et al. 2008].

As APs são estruturas de aprendizagem, que combinam abordagem pedagógica, inteligência artificial, educação a distância e concepção de tempo e espaço, e que são inspiradas em princípios das ideias construtivistas de Piaget e da pedagogia da pergunta de Freire, estabelecendo a Pedagogia da incerteza que implica em: (i) soluções de problemas reais, (ii) transformação de informações em conhecimento, (iii) autoria, expressão e interlocução, (iv) investigação e criação de novidades e (v) autonomia e cooperação [Carvalho et al. 2005].

Seguindo as teorias construtivistas e o conceito de Arquitetura Pedagógica, a CAPCOM foi modelada com o propósito de apoiar a construção colaborativa do conhecimento, seguindo a orientação das APs Colaborativas criadas pelo professor. Tal abordagem é implementada nas atividades com foco no tratamento colaborativo de multimídia, proporcionando a consolidação do conhecimento adquirido nas atividades de campo.

A forma como o sistema foi idealizado permite atender as necessidades de reflexão sobre uma aula de campo e seu respectivo objetivo pedagógico, atendendo tanto atividades conduzidas com crianças até atividades desenvolvidas com adultos, em aulas de campo relacionadas ao ensino de matemática, ciência (classificação, estruturação de conhecimento etc), engenharia, ciências biológicas, geografia, computação, por exemplo.

\section{Conceitos do MOrFEU usados na plataforma CAPCOM}

O projeto MOrFEu (Multi-Organizador Flexível de Espaços Virtuais) tem como objetivo libertar o professor da complexidade das tecnologias e ferramentas necessárias para sustentar as prática pedagógicas propostas pelo professor. Segundo [Menezes et al. 2008] o 
MOrFEu se pauta nos seguintes aspectos: plasticidade, ergonomia, redução da repetição de trabalho e redução da sobre carga cognitiva. Tais características são muito relevantes para a CAPCOM, dado que essa plataforma foi pensado para fomentar novas oportunidades de aprendizado em um contexto pós aula da campo.

Neste trabalho são usados três conceitos do MOrFEU: VCom (Veículo de Comunicação); UPI (Unidade de Produção Intelectual) e Template para VCom. Esses conceitos são usados na modelagem do CAPCOM, de modo a permitir ao professor flexibilidade para configurar os recursos digitais usados nas atividades colaborativas de consolidação do conhecimento desenvolvido na aula de campo, por meio da realização de operações cognitivas (colaborativas) sobre os textos multimídia elaborados no campo, tais como: classificação, criação de textos multimídia mais elaborados, etc.

Um VCom é um canal para troca de comunicações entre pessoas que nele assumem papéis, as vezes simultaneamente, de criadores e consumidores de conteúdo [Pereira 2015]. Alguns exemplos que materializam o conceito de VComs são: aplicativos de publicação de mensagem, blogs da internet, redes sociais digitais, revista, relatório de uma aula de campo, mapas mentais etc.

Segundo [Vieira 2011] "O conceito de UPI (unidade de produção intelectual) permite que todo texto (uma foto, um texto escrito, um vídeo, um áudio etc) seja registrado com autoria, data, versão etc, de modo independente de onde ela esteja publicada." Uma UPI é a unidade base de toda informação produzida, unidade essa que preserva a autoria e a característica de texto que essa unidade tem. Uma UPI pode assumir zero ou muitas associações com VComs. Como exemplo, em um VCom que se constitua em um relato de uma aula de campo, uma UPI pode ser um de seus componentes, em outro VCom que se constitua em um fórum, a mesma UPI pode ser uma das postagens e, em um terceiro VCom que se constitua em um mapa mental, a mesma UPI pode ser um dos elementos constituintes desse mapa. Tal flexibilidade pode ser configurada por uma ferramenta para construção de VCom, onde os critérios para a recepção e publicação de UPIs podem ser ajustados de acordo com a necessidade.

O conceito de Template para VCom permite configurar diferentes formas visuais para um mesmo conjunto de UPIs associados a um VCom. Segundo [Vieira 2011], "Cada VCom pode estar associado a diferentes templates, o que permite que cada usuário possa definir como deseja visualizá-lo." Como exemplo, um mapa mental, tipo de VCom, pode assumir vários arranjos, ao mesmo tempo.

Em um arranjo de mapa mental, todos os conceitos podem estar ligados de forma caótica e, em outro arranjo, as ligações entre os conceitos podem siguir uma orientação circular, no entorno do conceito central. Nesses exemplos, a UPI e o VCom continuam com a mesma estrutura, o que varia é apenas o aspecto visual, o Template do VCom.

\section{Arquitetura Pedagógica Colaborativa}

Atividades colaborativas são definidas como "[...]uma proposta pedagógica na qual estudantes ajudam-se no processo de aprendizagem, atuando como parceiros entre si e com o professor, com o objetivo de adquirir conhecimento sobre um dado objeto[...]" [Leite et al. 2005]

Para [Dillenbourg et al. 1995] aprender de forma colaborativa remete a uma 
situação na qual duas ou mais pessoas buscam aprender algo juntas, essa proposta é considerada muito relevante para apoiar o processo de aprendizado.

Aprendizagem de forma colaborativa requer um ambiente diferenciado, já que os professores e alunos desempenham novos papéis nessa abordagem. Os alunos se tornam elementos ativos e responsáveis por sua aprendizagem, nesse contexto o professor deixa de ser o único detentor do conhecimento e passa a promover as propostas pedagógicas, onde os alunos podem progredir com seus próprios esforços [Castro and Menezes 2011].

Usando a estrutura de AP definida por [Reinoso and Tavares 2015], para criar uma prática pedagógica colaborativa, é necessário definir os seguintes elementos: o objetivo pedagógico (o que aprender), atividades (o que fazer), método (como fazer) e recursos digitais (com que ferramentas).

O modelo de colaboração usado como referência pelo professor para criar cada AP colaborativa é o modelo 3C - comunicação, coordenação e cooperação [Pimentel et al. 2006].

A coordenação é desempenhada especialmente pelo professor que organiza os recursos, os participantes das tarefas e acompanha o desenvolvimento das atividades.

A comunicação é desempenhada por todos, mas os alunos, em especial, dependem da comunicação para colaborar na construção das atividades, para argumentação e negociação de compromissos.

A cooperação é desempenhada especialmente pelos alunos, seja nas atividades em grupo ou individuais, visto que mesmo nas atividades individuais existe a cooperação. Durante a cooperação os participantes podem processar e criar novos objetos.

Seguem três exemplos de Arquiteturas Pedagógicas criadas na plataforma CAPCOM (Construtor de AP para dinâmicas colaborativas com textos multimídia):

AP1: Arquitetura Pedagógica Cadeia Alimentar.

Contexto: Após uma aula de campo que acontece em um zoológico, o professor decide criar atividades pedagógicas na qual os alunos possam trabalhar com as mídias produzidas e registradas durante a aula, em um servidor (computador central), para aprender sobre a cadeia alimentar de um grupo de animais que dividem um mesmo ecossistema.

Objetivo pedagógico: - Aprender sobre os níveis tróficos de uma cadeia alimentar, dado um conjunto de seres vivos de um ecossistema.

Atividades: - Associar mídias produzidas em uma aula de campo, usando mapas mentais para registrar a associação.

Método: - O professor seleciona um conjunto de mídias que precisa ser explorado pelos alunos. O professor forma grupos de três alunos e solicita que os alunos colaborem para associar todas as mídias produzidos em uma aula de campo.

Recurso digital: - São usados recursos para edição de mapas mentais, suporte a grupos de colaboração e edição de UPIs.

Colaboração: - No recurso digital "edição de mapas mentais" os alunos trabalham com a fase de cooperação para construir o mapa mental. No recurso "suporte a grupos de colaboração" os alunos e o professor trabalham a fase de coordenação e 
comunicação para viabilizar os resultados a serem produzidos. No recurso "edição de UPIs" os alunos trabalham a fase de cooperação para compor as novas UPIs.

AP2: Arquitetura Pedagógica Relato de Grupo.

Contexto: Após uma aula de campo, que acontece em uma área industrial, o professor decide criar atividades pedagógicas colaborativas para os alunos relatarem sobre os conhecimentos adquiridos, usando as mídias produzidas e registradas durante a aula.

Objetivo pedagógico: - Criar relatórios colaborativos com as UPIs da aula em campo.

Atividades: - Combinar logicamente as UPIs (multimídia) e produzir um relatório das atividades desenvolvidas durante a aula de campo

Método: - O professor define que os alunos de um grupo podem acessar apenas os textos (UPIs) que produziram; forma grupos de cinco alunos e desafia o grupo a produzir um relatório das atividades conduzidas durante a aula de campo.

Recurso digital: - São utilizados recursos para edição de UPIs, suporte à colaboração e negociação para uso de UPIs.

Colaboração: - Com o recurso "edição de UPIs" os alunos trabalham a fase de cooperação para compor as novas UPIs. Com o recurso "suporte a grupos de colaboração" os alunos e o professor trabalham a fase de coordenação e comunicação para viabilizar os resultados a serem produzidos. Com o recurso digital "negociação de UPIs", os alunos e o professor trabalham a fase da comunicação, coordenação e cooperação em conjunto, para viabilizar o uso de uma mídia, de autoria de um aluno, por parte de outro aluno do mesmo grupo.

AP3: Arquitetura Pedagógica Fórum de Vídeos.

Contexto: Após uma aula de campo, onde um grupo de alunos de jornalismo entrevista pessoas que estão assistindo a uma peça de teatro, o professor decide criar atividades pedagógicas na qual os alunos possam apresentar e discutir sobre o processo de confecção dos vídeos registrados em cada entrevista durante a aula.

Objetivo pedagógico: - Aprender a registrar e usar vídeos Atividades - Combinar os vídeos de forma sequencial e registrar os vídeos

Método: - O professor define que a atividade será em grupo; define que só UPIs do tipo vídeo podem ser utilizadas; forma grupos de cinco alunos e desafia os alunos a se ajudarem para registrar seus vídeos de forma sequencial.

Recurso digital: - São usados recursos para edição de UPIs, suporte à colaboração, negociação para uso de UPIs e registro de novas UPIs

Colaboração: - Com o recurso digital "edição de UPIs" os alunos trabalham a fase de cooperação para compor as novas UPIs. Com o recurso digital "suporte a grupos de colaboração" os alunos e o professor trabalham a fase de coordenação e comunicação para viabilizar os resultados a serem produzidos. Com o recurso digital "negociação de UPIs", os alunos e o professor trabalham a fase da comunicação, coordenação e cooperação em conjunto, para viabilizar o uso de uma mídia, de autoria de um aluno, por parte de outro aluno de qualquer um dos grupos. Com o recurso digital "registro de UPIs" os alunos 
trabalham a fase de cooperação para enriquecer as possibilidades de vídeos que podem ser usados na execução da atividade.

\section{O Papel do Professor}

Durante as atividades, o professor deve produzir conflitos cognitivos entre os seus alunos, incentivando-os a pensar, a analisar quaisquer conteúdos, por meio do raciocínio lógico, da análise e da comparação [Giordani et al. 2006].

As interações entre os aprendizes podem ser afetadas por aspectos culturais diferentes como: estilos de comunicação; atitudes diante de conflitos; abordagens para executar uma tarefa; estilos de tomadas de decisões, atitudes diante de novas descobertas e abordagens sobre o conhecimento [Leite et al. 2005]. Por tanto a solução precisa atender as particularidade dos aprendizes, visando facilitar a interação.

Criando AP colaborativas, com o uso do CAPCOM, o professor ou mediador das atividades pedagógicas pode incentivar o trabalho colaborativo entre os alunos.

Como exemplo de uso do CAPCOM, o professor pode trabalhar o estudo sobre felinos, no contexto de uma aula de campo dentro de um zoológico, onde os alunos produzem textos multimídia com registros das observações que fizerem, e em um momento posterior à aula de campo, o professor pode propor o uso de uma AP (arquitetura pedagógica), com um Recurso Digital onde os alunos possam colaborar para construir relatos e consolidar conhecimentos sobre os felinos, a partir dos registros feitos por toda a turma.

Em outro exemplo, o professor pode criar uma AP, com a CAPCOM, para usar as multimídia produzidas pelos alunos em uma atividade de campo. Essa AP dispõe de Recurso Digital para os alunos construírem, de modo colaborativo, um relato descritivo das atividades executadas, incluindo mídias do tipo foto, foto esférica, vídeo e áudio.

As mídias do relato podem fazer associações entre os conceitos apreendidos durante a visita à atividade e os mídias (UPIs) produzidos, de modo a criar uma classificação (taxonomia) dessas mídias.

[Ocepek et al. 2013] defende que alunos e professores, com diferentes estilos de aprendizagem, tendem a preferir determinadas mídias em detrimento a outras, de acordo com o estilo de aprendizagem. Nesse sentido o professor precisa incentivar os alunos a registrarem as informações de modo colaborativo, podendo os registros produzidos atenderem aos estilos de aprendizagem de cada aluno.

Antes da atividade de campo e após essa atividade, o professor pode promover o debates sobre o propósito pedagógico das atividades, apresentando informações que possam ser verificadas durante a atividade, estimulando nos alunos o aprendizado colaborativo e o aprendizado por associação.

\section{A plataforma CAPCOM}

A plataforma CAPCOM usa UPIs criadas em atividades de campo, por uma turma, para a criação de arquiteturas pedagógicas que propõem atividades pedagógicas de consolidação do conhecimento adquirido na atividade em campo. Essas arquiteturas pedagógicas são usadas pelos aprendizes em momento posterior às atividades de campo. 
Segundo [Vieira and Tavares 2015] novas práticas de ensino-aprendizagem sustentadas pelas arquiteturas pedagógicas sugerem plataformas tecnológicas sintonizadas às necessidades de interação sob protocolos distintos. Os ambientes colaborativos, principalmente os ambientes virtuais de aprendizagem, devem apresentar ao professor, enquanto coordenador das atividades, recursos funcionais capazes de tornar possível a implementação das suas estratégias de ensino e aprendizagem.

Aulas de campo são atividades que permitem reflexão sobre os conhecimentos articulados em estudos teóricos, as atividades realizadas em campo geram dados que são organizados em relatórios. A plataforma AM [Pereira et al. 2015] permite o registro de UPIs (textos) durante a realização das atividades de campo, mas carece de suporte para a reflexão sobre esses textos elaborados no campo, em momento posterior à aula de campo.

Essa pesquisa propõe a plataforma CAPCOM com recursos digitais que suportem atividades de reflexão sobre as UPIs criadas em campo, de modo a permitir novas oportunidades de aprendizagem. Entre as atividades citadas estão: consultar, manipular e combinar, em diferentes modos definidos pelo professor, os registros (UPIs) produzidos em aulas de campo que tenham sido feitos com o uso dos recursos da plataforma AM. Sendo assim, a plataforma CAPCOM permite a criação de arquiteturas pedagógicas com atividades que são complementares e posteriores às atividades realizadas com a plataforma AM.

A plataforma AM [Pereira et al. 2015] fornece recursos digitais para suporte às atividades pedagógicas em aulas de campo. Com ela, um educador pode delimitar a área onde a atividade de campo será realizada, definir subáreas onde informações diferenciadas podem ser acessadas, definir regras de comportamento para os alunos em cada uma das subáreas, acompanhar o trajeto de cada aluno da turma e etc.

Cada aluno, por sua vez, com o uso de um dispositivo móvel, pode criar registros multimídia locativos (UPILocs) ao longo do trajeto que realizar, acessar as informações disponíveis em cada subárea que estiver visitando, interagir com colegas e etc.

A plataforma CAPCOM - Construtor de Arquiteturas Pedagógicas para Dinâmicas Colaborativas com Multimídia dispõe de uma Biblioteca de Recursos Digitais. A Figura 1 apresenta os componentes da plataforma CAPCOM. Também apresenta os atores importantes no cenário de uso dessa plataforma: o professor que usa a CAPCOM para criar as APs, configurar os recursos digitais e visitar os textos (UPIs), produzidos pelos alunos; os alunos que usam os recursos digitais para desenvolverem colaborativamente as atividades propostas em cada AP e criarem novos textos multimídia (UPIs) e os visitantes que usam os recursos digitais para visualizarem os textos (UPIs) produzidos.

Para criar uma Arquitetura Pedagógica (AP) na plataforma CAPCOM, o professor descreve o objetivo pedagógico, a atividade pedagógica e a metodologia, em texto escrito. Depois de cadastrar essas informações, o professor seleciona e configura os recursos disponíveis que vão compor os Recursos Digitais da AP, podendo selecionar mapa mental, composição de mídias, grupos de usuários, classificação de UPI etc.

O professor usa o CAPCOM para construir arquiteturas pedagógicas colaborativas. Os aprendizes executam cada AP (arquitetura pedagógica), usando cada recurso digital alocado pelo professor na AP, para desenvolverem as atividades pedagógicas propostas. 


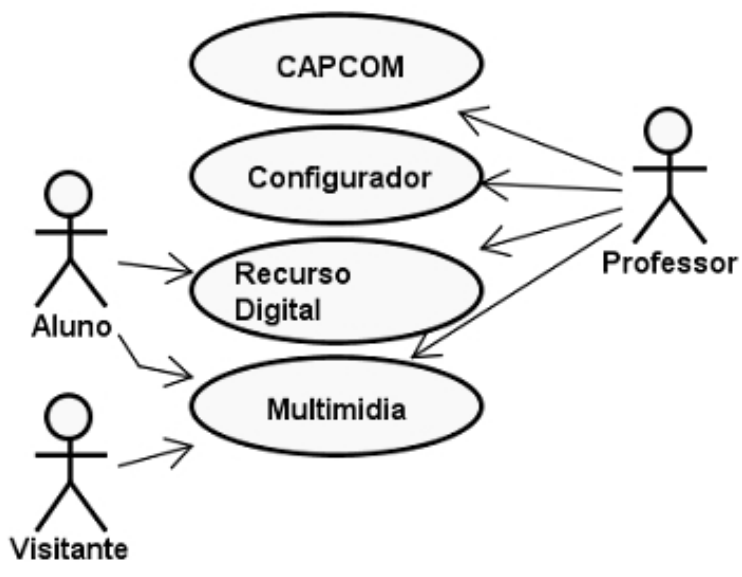

Figura 1. Diagrama de casos de uso para a CAPCOM.

A CAPCOM permite que o professor configure os Recursos Digitais para cada AP criada por ele, atendendo as necessidades específicas de uma aula de campo.

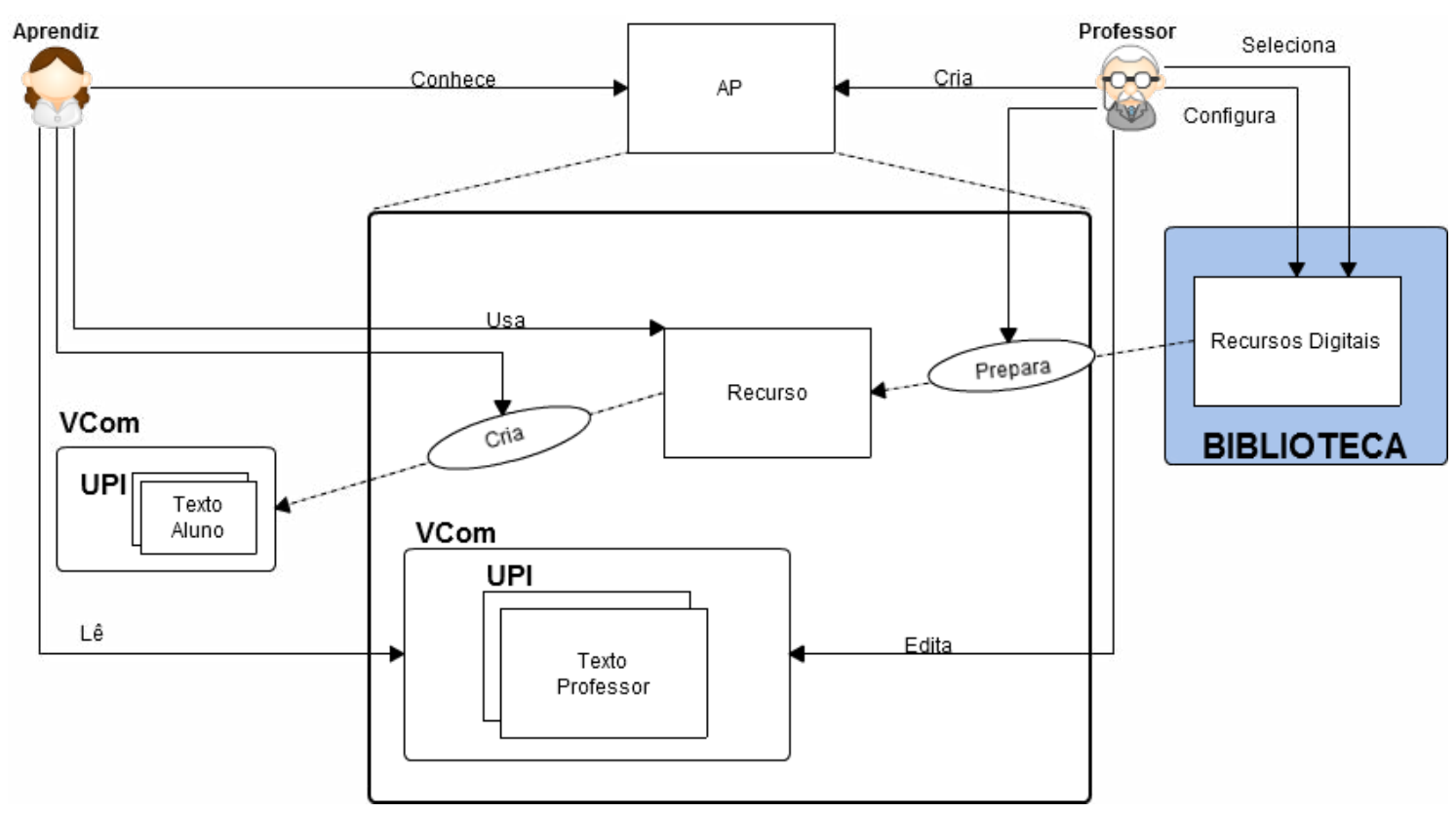

Figura 2. Arquitetura e funcionamento da plataforma CAPCOM.

A Figura 2 apresenta a arquitetura da plataforma CAPCOM. A CAPCOM permite que o professor configure cada Recurso Digital de uma AP e define as regras e a forma da atividade - VCom. Já os alunos podem processar os registros produzidos na aula de campo - UPI - na atividade proposta pelo professor, de modo colaborativo, e modificar o aspecto visual do conteúdo produzido na atividade - Template de VCom - também colaborativamente.

A CAPCOM implementa diversos editores que permitem a manipulação de UPIs para compor novas UPIs (UPIs compostas) ou a criação de VComs (VCLocs) ou templates, de modo a permitir novas formas de organizar e apresentar as UPIs criadas na aula de campo. Tudo isso em atividades realizadas após a aula de campo. 
Tabela 1. Distribuição e uso dos recursos digitais da biblioteca do CAPCOM.

\begin{tabular}{|l|l|l|l|}
\hline \multirow{2}{*}{ Recursos } & \multicolumn{3}{|c|}{ Arquiteturas Pedagógicas } \\
\cline { 2 - 4 } & AP1 & AP2 & AP3 \\
\hline Edição de mapas mentais & Sim & Sim & Sim \\
\hline Suporte a grupos de colaboração & Sim & Sim & Sim \\
\hline Edição de UPIs & Sim & Não & Sim \\
\hline Negociação de UPIs & Não & Sim & Não \\
\hline Registro de UPIs & Não & Não & Sim \\
\hline
\end{tabular}

Baseadas nas APs apresentadas na seção 5, foram levantados os seguintes recursos digitais que compõem a biblioteca da CAPCOM:

1. Edição de mapas mentais

2. Suporte a grupos de colaboração

3. Edição de UPIs

4. Negociação de UPIs

5. Registro de UPIs

A Tabela 1 apresenta a distribuição e uso dos recursos da biblioteca da plataforma CAPCOM para criação e uso das APs.

Usando as UPIs de uma aula de campo, o professor pode definir:

- regras sobre o uso delas (um aluno pode usar apenas suas UPIs ou pode usar UPIs de colegas);

- grupos de usuários para colaborar no uso dessas UPIs (textos em múltiplas mídias, como fotos, textos escritos, vídeos, áudios etc) e na criação de novas UPIs compostas;

- a atividade como individual e habilitar a colaboração apenas em outra fase da atividade;

- se a AP terá como foco a composição de novas UPIs ou a classificação das UPIs criadas.

Os recursos digitais configurados pelo professor para cada arquitetura pedagógica, permitem ao aluno articular as UPIs que foram criadas durante a aula de campo, de modo a produzir novos registros, a partir da associação dessas UPIs de diversas formas.

O resultado da manipulação das UPIs pelo aluno fica disponível em outra parte da CAPCOM, onde o professor e o aluno podem ter acesso. A Figura 3 apresenta um exemplo de UPI criada por recurso digital da plataforma CAPCOM, onde é incorporado um menu para interligar a UPI da figura com outras UPIs.

\section{Trabalhos Correlatos}

A Tabela 2 apresenta uma comparação da plataforma CAPCOM com outros sistemas que também têm o objetivo de ajudar o educador a criar atividades associadas a sua prática pedagógica colaborativa.

Na coluna "Projetado para" é apresentado o(s) modo(s) de uso do sistema: "Móvel" significa que o sistema foi desenvolvido para usar recursos de dispositivos 


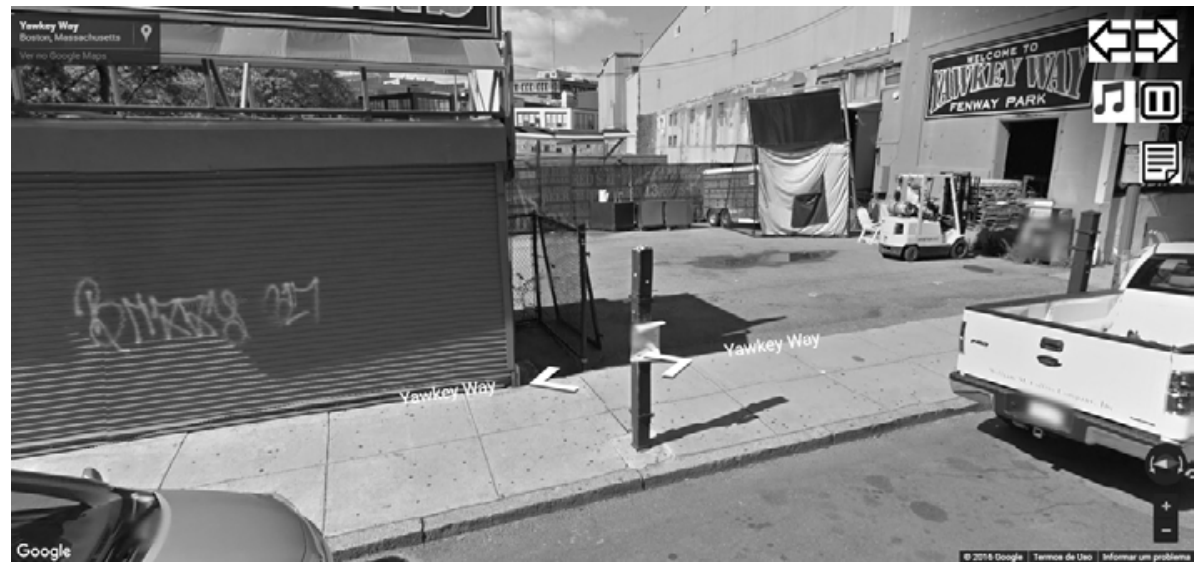

Figura 3. Um exemplo de UPI gerada em uma AP.

móveis e "Web" indica que o sistema foi construído para usar recursos da Web. A coluna "Principal Uso" apresenta o propósito principal de construção do sistema. A coluna "Colaboração" define se o sistema suporta a colaboração durante a utilização, dentre as diversas possibilidades oferecidas. A coluna "Atividade" indica se o sistema permite a criação de atividades pedagógicas.

Tabela 2. Comparação entre sistemas digitais para atividades pedagógicas.

$\begin{array}{lllll}\text { Sistema } & \text { Projetado para } & \text { Principal uso } & \text { Colaboração } & \text { Atividades } \\ \text { MOrFEu } & \text { Web } & \text { Educacional } & \text { Sim } & \text { Sim } \\ \text { Aris Games } & \text { Móvel } & \text { Jogos } & \text { Não } & \text { Sim } \\ \text { Youubi } & \text { Móvel } & \text { Educacional } & \text { Sim } & \text { Sim } \\ \text { Plataforma CAPCOM } & \text { Móvel e Web } & \text { Educacional } & \text { Sim } & \text { Sim }\end{array}$

A plataforma Youubi, assim como a plataforma CAPCOM, também é projetada para dispositivos móveis, tem suporte à locatividade, tem suporte a colaboração durante as atividades e é voltada para Aplicações Educacionais. Entretanto, na plataforma Youubi as atividades pedagógicas são pré-definidas, o professor não pode ajustar a atividade às necessidades pedagógicas da turma. Já o CAPCOM permite que o professor crie as atividades de acordo com sua proposta pedagógica.

\section{Considerações Finais}

De modo complementar à proposta do AM, este trabalho apresentou a plataforma CAPCOM (Construtor de Arquiteturas Pedagógicas Com textos Multimídia), que permite a criação e uso de arquiteturas pedagógicas (APs) para a realização de atividades de aprendizagem colaborativa, com o uso de textos multimídia (UPIs), produzidos durante atividades de campo.

O uso das APs proporciona novas oportunidades de aprendizado, aproveitando o potencial pedagógico dos registros (UPIs) produzidos e armazenados nas atividades de campo, com o uso da plataforma AM (Aprendizagem em Movimento) [Pereira et al. 2015].

A pataforma CAPCOM foi criada para completar o ciclo pedagógico de uma aula de campo que é constituído de: planejamento da aula de campo, a aula propriamente 
dita e as atividades de reflexão sobre os dados coletados em campo. A plataforma CAP$\mathrm{COM}$ fornece recursos para criar, configurar e usar arquiteturas pedagógicas que tratem de atividades posteriores à aula de campo.

$\mathrm{O}$ artigo apresentou três exemplos de arquiteturas pedagógicas criadas pela plataforma CAPCOM e contextualizou o uso de cada uma dessas APs, bem como, descreveu alguns aspectos da dinâmica colaborativa entre os alunos realizadas durante o uso de cada AP.

Em continuidade à pesquisa descrita aqui, pretende-se ampliar o uso dos recursos digitais da CAPCOM em dispositivos móveis com iOS e Windows Phone. Também pretende-se ampliar as possibilidades de textos que a plataforma pode trabalhar, incluindo vídeos esféricos e outras mídias ainda pouco exploradas, mas que têm grande potencial para o propósito pedagógico.

\section{Referências}

Carvalho, M. J. S., Nevado, R. A. d., and Menezes, C. d. (2005). Arquiteturas pedagógicas para educação a distância: concepções e suporte telemático. Anais do XVI Simpósio Brasileiro de Informática na Educação, Juiz de Fora-MG. Brasil, pages 351-360.

Castro, A. and Menezes, C. (2011). Aprendizagem colaborativa com suporte computacional. Sistemas Colaborativos, pages 135-153.

Chagas, I. (2002). Trabalho colaborativo condição necessária para a sustentabilidade das redes de aprendizagem. Redes de aprendizagem. Redes de conhecimento. Lisboa: Conselho Nacional de Educação, pages 71-82.

Dillenbourg, P., Baker, M. J., Blaye, A., and O’Malley, C. (1995). The evolution of research on collaborative learning. Learning in Humans and Machine: Towards an interdisciplinary learning science., pages 189-211.

Giordani, A. C. C., Audino, D. F., and Cassol, R. (2006). Inserção do google earth no ensino de geografia. IN: Jornada da Educação, UNIFRA, Centro Universitário Franciscano, Santa Maria/RS, pages 1-8.

Lei, P.-L., Kao, G. Y.-M., Lin, S. S., and Sun, C.-T. (2009). Impacts of geographical knowledge, spatial ability and environmental cognition on image searches supported by gis software. Computers in Human Behavior, 25(6):1270-1279.

Leite, C. L. K., PASSOS, M. d. A., Torres, P. L., and Alcântara, P. R. (2005). A aprendizagem colaborativa na educação a distância on-line. In CONGRESSO INTERNACIONAL DE EDUCAÇÃO A DISTẨNCIA, volume 12, pages 1-10.

Menezes, C. S., de Nevado, R. A., de Castro Jr, A. N., and Santos, L. N. (2008). Morfeumulti-organizador flexível de espaços virtuais para apoiar a inovação pedagógica em ead. In Anais do Simpósio Brasileiro de Informática na Educação, volume 1, pages 451-460.

Ocepek, U., Bosnić, Z., Šerbec, I. N., and Rugelj, J. (2013). Exploring the relation between learning style models and preferred multimedia types. Computers \& Education, 69:343-355. 
Oliveira, A. and Correia, M. D. (2013). Aula de campo como mecanismo facilitador do ensino-aprendizagem sobre os ecossistemas recifais em alagoas. Alexandria Revista de Educação em Ciência e Tecnologia, 6(2):163-190.

Pereira, B., Almeida, R., Tavares, O., and de Menezes, C. (2015). Am: Uma plataforma digital para criacao e uso de veículos de comunicacao locativos para atividades pedagogicas. In Anais do Simpósio Brasileiro de Informática na Educação, volume 26, pages 131-139.

Pereira, B. C. (2015). Aprendizagem em movimento - uma plataforma para a criaçção e uso de veiculos de comunicação na realização de arquiteturas pedagogicas. Master's thesis, Programa de pós graduação em em Informática da UFES, Vitória-ES.

Pimentel, M., Gerosa, M. A., Filippo, D., Raposo, A., Fuks, H., and Lucena, C. J. P. (2006). Modelo 3c de colaboração para o desenvolvimento de sistemas colaborativos. Anais do III Simpósio Brasileiro de Sistemas Colaborativos, pages 58-67.

Reinoso, L. and Tavares, O. (2015). Mvlibras: ambiente digital para comunidades de aprendizagem com recursos inclusivos para surdos. In Anais do Simpósio Brasileiro de Informática na Educação, volume 26, pages 772-781.

Seniciato, T. and Cavassan, O. (2004). Aulas de campo em ambientes naturais e aprendizagem em ciências-um estudo com alunos do ensino fundamental field classes in natural environment and science learning-a study with students from elementary school. Ciência \& Educação, 10(1):133-147.

Vieira, R. R. M. (2011). Uma arquitetura de software para o morfeu: Apoiando a realização de arquiteturas pedagógicas em espaços virtuais colaborativos. Master's thesis, Dissertação (Mestrado em Informática)-UFES, Vitória-ES, Agosto.

Vieira, R. R. M., dos Santos, O. L., do Prado Rafalski, J., Bada, E. M., da Silva, H. F. A., and de Menezes, C. S. (2011). Coordenaçao nas atividades colaborativas em ambientes de aprendizagem-uma avaliaçao na implementaçao de arquiteturas pedagógicas. RENOTE, 9(1).

Vieira, R. R. M. and Tavares, O. d. L. (2015). Modelo para descrição de espaços colaborativos: Apoiando a realização de arquiteturas pedagógicas. Sistemas Colaborativos, pages $189-196$. 